\title{
Enfoques preventivos del uso y abuso de cannabis y problemas asociados
}

\author{
Amengual Munar, M. \\ Cap dels Serveis de Joventut. \\ Conselleria de Benestar Social. Govern de les Illes Balears
}

Correspondencia: Miquel Amengual Munar. PI. Obispo Berenguer de Palou, 10. 07003 Palma de Mallorca

Tel. 971177440 Fax 971176467 E-mail: ameng004@correo.cop.es

\section{Resumen}

Del análisis de los datos disponibles se desprende que, como criterios generales preventivos: 1) la prevención del consumo de cannabis no puede plantearse de manera aislada a la del consumo de otras drogas; 2) la prevención debe incluir como primer objetivo la reducción del nivel de consumo de alcohol y tabaco; 3) es necesaria la reducción de la disponibilidad de cannabis, combatir la tolerancia social y familiar hacia su consumo, y contrarrestar la presión cultural y económica que sostiene y estimula el consumo; 4) en el nivel de las personas y grupos es necesario reforzar las competencias y habilidades para hacer frente a las presiones a favor del consumo, cambiar la percepción normativa y la percepción del riesgo respecto del consumo de cannabis.

Se analizan diversos programas e intervenciones, y revisiones de programas, con los siguientes resultados: a) los programas universales más eficaces son los que no se centran de manera exclusiva en el cannabis y que, además, apuntan a diversos componentes de la conducta, mientras que aquellos que se basan exclusivamente en la transmisión de información no reducen el consumo ni las prácticas de riesgo; b) no existen prácticamente programas selectivos, o dirigidos a grupos de riesgo, específicos sobre cannabis. En general, los programas existentes adolecen de la necesaria planificación, aparecen como respuesta inmediata a necesidades puntuales y no siguen ningún procedimiento de evaluación fiable

Palabras clave: Prevención, cannabis, programas preventivos, evaluación, revisión.

\section{Summary}

Analysis of the data leads to the following preventive propositions: 1) the prevention of cannabis use cannot be approached normally isolated from other drugs prevention; 2 ) a reduction in the level of alcohol and tobacco consumption must be included as a primary objective of cannabis prevention; 3 ) it could be useful a reduction in cannabis availability, combating social and family tolerance and counteracting the cultural and economical pressures that sustain and stimulate its use; 4) at the level of people and groups its important reinforcing abilities and skills to face up to the pressures, changing the social perception that facilitates its use.

The most effective 'universal' prevention programmes are those that do not focus exclusively on cannabis and which, in addition, are aimed at diverse components of behaviour whereas those that are based exclusively on the provision of information do not reduce use or harmful practices; there are few 'selective' programmes directed at groups at risk that concentrate specifically on cannabis and, in addition, they do not focus on the drug itself but on the modification of a number of risk factors. In general terms all this prevention programmes lack the necessary planning and evaluation.

Key words: prevention, cannabis, programmes, evaluation, review. 


\section{INTRODUCCIÓN}

E I cánnabis es la sustancia ilegal más frecuentemente utilizada en los países occidentales. En Estados Unidos, según los datos de 1997 (NIDA, 1999), el $80 \%$ aproximadamente de los consumidores de drogas ilegales usan derivados del cánnabis, siendo los varones de entre 18 y 25 años los que tienen mayores tasas de consumo. En aquel país, entre 1996 y 1997, la tasa de incremento del consumo fue considerablemente menor que la que se observó en años anteriores. Además, por primera vez en la década se registró un incremento en el grado de desaprobación de la marihuana entre los jóvenes, pese a que no disminuyó su nivel de disponibilidad y facilidad de obtención. Sin embargo, hay datos que obligan a moderar el optimismo: aumentaron las intervenciones de urgencia relacionadas con los efectos adversos del uso de los derivados del cánnabis, creció el porcentaje de inicios de tratamiento por uso de cánnabis, y esta sustancia ya supera a la cocaína como droga más detectada en orina entre los detenidos por cualquier causa.

En Europa la situación es parecida. El informe de 1999 del Observatorio Europeo de las Drogas y las Toxicomanías indica que el cánnabis es la sustancia ilegal más frecuentemente utilizada en todos los países (EMCDDA, 1999). Pese a las diferencias entre países, se observa una tendencia a la convergencia: en los países de prevalencia más elevada, el consumo tiende a estabilizarse o a descender, mientras que en los de prevalencia más baja, el nivel de consumo está creciendo. Por otra parte, el consumo de cánnabis no está vinculado a ningún contexto específico social o de ocio, ni a ningún grupo concreto, lo que da idea del carácter de normalidad de esta práctica y de su nivel de penetración en nuestra sociedad. Según los diferentes países, alcanza porcentajes de utilización del 10-30 \% entre los adultos, del 5$40 \%$ entre los jóvenes de 15 a 16 años, y del 16-40\% entre los jóvenes de alrededor de 25 años. Además, aunque la proporción de admi- siones a tratamiento más elevada sigue siendo la correspondiente a los opiáceos, está disminuyendo al tiempo que aumenta en algunos países la demanda de tratamiento por cánnabis, que es la principal droga de consumo del 2-10\% de pacientes, sobre todo en los consumidores más jóvenes.

En España, las últimas encuestas de ámbito estatal disponibles indican que el cánnabis sigue siendo la droga ilegal de consumo más extendido. Según la Encuesta Domiciliaria de 1997 aproximadamente uno de cada cinco españoles ha probado estas sustancias $(21,7 \%)$. Se observa un incremento respecto de 1995 y, aunque esto no signifique necesariamente que asistimos a un aumento del consumo en la población general, parece que se confirma la expansión en la población juvenil y adolescente que evidenció la Encuesta Escolar de 1996. En esta población se incrementan todos los indicadores de consumo que alcanzan un $15 \%$ en el último año y un $7 \%$ en el último mes. Un $2,6 \%$ de los españoles de 15-65 años puede considerarse que son consumidores habituales de cánnabis (Observatorio Español sobre Drogas, 1999).

En una línea concordante son los datos del estudio comparativo de la vida nocturna y uso recreativo de drogas en diversas ciudades europeas (Calafat y otros, 1999). Según este estudio, el $11,3 \%$ de los jóvenes utilizan cánnabis varias veces a la semana, y un $10 \%$ lo consumen cada día. Cuando se consume más de una sustancia tóxica, en el 88,1\% de las combinaciones está presente el cánnabis, y la mitad de los policonsumos incluyen alcohol y cánnabis. Además, el $24,2 \%$ de los usuarios de cánnabis creen que les sería muy difícil renunciar a su consumo.

La situación descrita no apunta ninguna señal de cambio significativo, por lo menos en el sentido de que a medio plazo se produzca una reducción sensible del consumo. Sin duda, las drogas satisfacen una necesidad social para la que nuestra sociedad no dispone ningún otro producto ni actividad alternativos. Sólo cabe plantearse su reducción en la medida en que seamos capaces de generar un cambio importante en las formas de satisfacer 
las necesidades personales y sociales de una parte cada vez mayor de la población, lo que nos introduce de lleno en la prevención.

Esta es una tarea tanto más urgente por cuanto de cada vez más se confirman las sospechas de los efectos del THC sobre diversas estructuras cerebrales, sobre el sistema endocrino y sobre el sistema inmunológico, con las consiguientes repercusiones sobre las funciones cognitivas, de memoria, manejo de emociones y afectos, sistema motivacional y de conducta en general, y su inevitable impacto en el nivel de desempeño en diversos aspectos de la vida individual, familiar, comunitaria y social.

\section{REQUISITOS GENERALES DE LA PREVENCIÓN}

\subsection{La prevención como tarea sistemati- zada.}

El significado corriente de la palabra prevención es bastante claro. Se trata de una opinión, generalmente desfavorable, acerca de alguien o algo. En consecuencia, prevenir es informar o avisar de un riesgo, con la intención de influir o predisponer a una persona contra algo.

Esta palabra, trasladada sin más al discurso político, técnico e incluso científico, cuando se refiere al consumo de drogas, se convierte en uno de los comodines y recursos más ambiguos que se conocen. En su aplicación práctica, eso que se llama hacer prevención, puede llegar a significar casi cualquier cosa. Demasiado a menudo se conserva su significación popular y se identifican prevención e información, a pesar de que hay sobradas evidencias de que la información, siendo un componente importante de la prevención, no agota todo su significado. Por eso no es extraño que, incluso en reuniones de expertos sobre drogas, se puedan escuchar manifestaciones de desconfianza hacia la prevención u opiniones como que la prevención no debe hacerse a edades muy tempranas por- que podrían incitar al consumo. Tales manifestaciones denotan un manejo del concepto de prevención totalmente ajeno al discurso preventivo, y un asombroso desconocimiento de los avances hechos en este campo durante los últimos veinte años.

Porque también puede haber un discurso científico de la prevención. Para ese discurso, la prevención es un concepto que va más allá del significado común de esta palabra o, por lo menos, no se limita a su concepción puramente informativa.

Ya en 1981 el grupo de expertos reunidos por el Comité Europeo de Salud Pública se afirmaba en su informe final: "una acción de prevención primaria tanto en lo que concierne a la educación para la salud como a la intervención en el ecosistema, necesita un conocimiento apropiado de los elementos etiológicos: es decir, los factores de riesgo y los factores para la defensa y protección. Exige igualmente un conocimiento y una verificación experimental de la metodología en prevención primaria. En tercer lugar, toda intervención implica igualmente un procedimiento de evaluación permanente" (Comité Européen de Santé Publique, 1981)

Cuando hablamos de prevención nos referimos, por tanto, a un conjunto sistemático y organizado de actuaciones tendentes a evitar o reducir la manifestación de un problema o de sus consecuencias.

Esta sistematización requiere:

a) Una definición del problema que se quiere prevenir, de su dimensión y de las personas que están expuestas a padecerlo. De esta manera podrán establecerse las metas, es decir, los objetivos generales o efectos (outputs) genéricos de la prevención que se va a emprender, los recursos y servicios que se deberán movilizar o implementar, las poblaciones destinatarias y los indicadores para la evaluación.

b) Un marco teórico que dé cuenta de los factores que contribuyen o evitan la aparición del problema definido, y de los mecanismos que favorecen o que disminuyen el riesgo. De esta manera se podrán definir 
los cambios específicos que se van a perseguir y en qué grupos determinados de la población, y mediante qué organización de servicios.

c) Una estrategia de intervención, es decir, un conjunto de acciones que modifiquen aquellos factores, lo que también implica un conocimiento de los procedimientos a través de los cuales es posible producir algún cambio favorable en los comportamientos individuales y sociales determinantes del problema. De esta manera podremos determinar quienes van a intervenir, la secuencia de actividades que han de llevar a cabo y los objetivos operativos o resultados (outcomes) concretos que se han de producir con cada una de ellas.

d) Finalmente, la evaluación. Porque, al hablar de prevención hablamos de tentativas más o menos logradas, ya que la intencionalidad preventiva no se verifica en todos los casos. La eficacia preventiva de un programa sólo puede establecerse con posterioridad, a través de una cuidadosa evaluación. Esta evaluación permitirá, en primer lugar, comprobar si se han conseguido los resultados esperados; en segundo lugar, comprobar si la estrategia diseñada, las técnicas utilizadas, los mediadores implicados, etc. son útiles para la prevención; finalmente, comprobar si la teoría que habíamos elaborado, y en la que se basaba el programa, tiene algún valor.

\subsection{La necesidad de planificación.}

Los cuatro pasos que se acaban de señalar, recogen los rudimentos de la planificación.

La planificación no es sólo una quimera de mentes cuadriculadas y personalidades obsesivas, cuya finalidad es acabar con lo que tiene de vivo la experiencia. Es cierto que en algunos casos la planificación puede limitar y anular la capacidad de intervención y la creatividad, y que a veces substituye la intervención o la retrasa, y se convierte en una excusa para no hacer nada. Pero cualquier actividad requiere un mínimo de planificación, ya sea consciente o inconsciente.
La falta de planificación en lo que concierne a la prevención lleva a confundir los niveles, a perseguir objetivos poco realistas, al fracaso y al descrédito general de la prevención.

No hay una prevención única y general. Aunque puede haber una finalidad última para la prevención que pueda ser compartida por los distintos enfoques, como es la promoción de estilos de vida y comportamientos saludables, lo que esto signifique también puede ser muy variable. No es lo mismo prevenir el inicio que intentar un uso razonable o evitar consecuencias adversas del consumo de drogas. Cada uno de estos objetivos se dirige a individuos diferentes, en contextos distintos y con métodos diferentes.

En un segundo nivel, también es importante distinguir, por ejemplo, entre comportamientos de inicio o que pueden llevar al inicio del consumo, uso ocasional de drogas, uso regular o frecuente y abuso de drogas. Cada una de estas conductas se establece a través de distintos caminos, genera dinámicas particulares en el sujeto y en su entorno, puede tener consecuencias diferentes y requiere estrategias de intervención específicas, por lo que es necesario adoptar unos objetivos claros que orienten nuestra intervención.

La prevención no significará nada si no se define qué es lo que se quiere prevenir. Pero ello requiere una gradación de objetivos. Por eso es importante establecer unas metas que orientarán la tarea. Pero estas grandes metas no pueden ser los objetivos de los programas ni de la intervención.

\subsection{Metas generales.}

La mayoría de los programas preventivos asumen al menos una de las cinco concepciones clásicas de lo que debe perseguirse con la prevención (Hawkins, Lishner y Catalano, 1985):

1. Lo que se debe prevenir es el abuso de drogas, entendido como patrón de uso patológico. La definición de uso patológico se basa en distintos criterios, como, por ejemplo, los que establece el DSM-IV. 
2. Un segundo tipo de conducta que se podría prevenir es el uso regular de drogas, independientemente de si este uso es o no causa de otros problemas en las distintas áreas de desempeño del sujeto. Este planteamiento se justifica por las consecuencias desfavorables para la salud que se ha podido comprobar que tiene el uso de determinadas drogas. De ello también se deriva que la intervención preventiva sólo estará justificada en la medida en que el tipo de uso que se quiere evitar es una causa conocida de un problema de salud.

3. Desde otro punto de vista, podemos intentar prevenir cualquier uso de drogas en la adolescencia, y cualquiera que sea su frecuencia e independientemente de los problemas que ocasione. Es un objetivo razonable si entendemos que el uso de drogas en la adolescencia puede tener un impacto negativo en los procesos de cambio que caracterizan este período. Además, el riesgo de uso de drogas parece incrementarse durante la adolescencia y no se puede descartar que para muchos adolescentes la experimentación con drogas cumpla algún papel, en unos casos favoreciendo y en otros estorbando su tarea de separación del adulto y formación de su identidad. Sin embargo, la meta de prevenir el uso adolescente de drogas es demasiado ambigua y puede llevar a planteamientos bastante opuestos, por lo que debe matizarse mediante otras consideraciones que permitan determinar los objetivos y las estrategias. Por ejemplo, según el tipo de droga y las consecuencias que se le atribuyan, sería posible preconizar tanto el uso responsable como la abstinencia de drogas.

4. Otra finalidad posible es retrasar la edad de inicio. Parece una meta limitada que, además, da por supuesto que el uso de drogas es inevitable. Sin embargo, es un planteamiento realista y de gran importancia preventiva. Según se desprende de los estudios epidemiológicos, el inicio tempra- no del consumo de una droga es uno de los mejores predictores del abuso posterior de esta y de otras drogas. Existe abundante información sobre este punto a partir de los estudios clásicos de Kandel (1978, 1982). De hecho, hay acuerdo en considerar que cuanto antes se inicie el consumo será más difícil mantenerlo dentro de unos márgenes razonables. Por otra parte, la mayor implicación en el consumo de una droga se convierte en un factor de riesgo para el uso de otras drogas, lo que nos lleva al quinto tipo de objetivos.

5. Un grupo interesante de objetivos es el que considera prioritaria la prevención de drogas específicas y, de manera más concreta, plantea centrarse en algunas drogas como el tabaco, el alcohol y los derivados del cánnabis, unánimemente consideradas substancias de inicio al consumo de otras drogas. Se ha comprobado que el uso de una droga habitualmente se establece a partir de un determinado consumo previo de otra substancia. Así, el consumo de alcohol o de tabaco precede al consumo de derivados de cánnabis, los cuales a su vez preceden el consumo de otras drogas ilegales (Kandel, 1978). Además, cuanto mayor sea el consumo de alcohol o de tabaco, mayor será la probabilidad de consumo de cánnabis; de la misma manera, cuanto mayor sea el consumo de cánnabis, también será mayor la probabilidad de consumo de otra droga del siguiente nivel, etc. (Calafat, Amengual, Farrés y Monserrat, 1983; Calafat, Amengual, Mejías y Borrás, 1989; Amengual, Calafat y Palmer, 1993). En cualquier caso, es importante elegir cuál será la droga (o drogas) sobre la que se centrarán los esfuerzos preventivos, y decidir si el objetivo será retrasar su inicio, la experimentación, el uso regular o el abuso.

Posteriormente Hawkins y colaboradores ${ }^{1}$ han añadido dos nuevos objetivos posibles.

6. Los esfuerzos preventivos deberían dirigirse a controlar las circunstancias que impli-

Citado por Gilchrist (1991). 
can un riesgo personal o de terceras personas como consecuencia del consumo de drogas. Se citan, como ejemplo, conducir o mantener relaciones sexuales bajo los efectos del alcohol. Se trata, en definitiva, de evitar las conductas dañinas que se pueden producir bajo los efectos de las drogas, o de minimizar algunos de los posibles efectos adversos del consumo.

7. Finalmente, otra de las metas será comenzar la prevención cuanto antes, en la primera infancia, para neutralizar los precursores del consumo. No obstante, los objetivos específicos que se desprenden de este enfoque todavía no se sustentan en evidencias empíricas, sino más bien en modelos teóricos que establecen relaciones entre determinadas situaciones psicológicas o sociales, algunos comportamientos inadaptados y el consumo de drogas.

Parece evidente que la adopción de unas u otras de estas metas guiará tanto el tipo de estudios etiológicos que se emprendan para definir los objetivos específicos, como para la selección de los destinatarios y de las estrategias de la prevención.

\subsection{Para definir los objetivos.}

Establecidas las metas, es necesario definir unos objetivos precisos.

Mientras que las metas pueden entenderse como objetivos generales o efectos genéricos que debe perseguir la prevención, los objetivos específicos son aquellos efectos que deben alcanzarse como resultado de un proceso planificado de intervenciones, en unas situaciones concretas de necesidad que se particularizan en unos colectivos y momentos bien definidos. A su vez, cada intervención deberá alcanzar unos resultados inmediatos, acordes con los objetivos específicos.

Los objetivos se suelen definir a partir de observaciones de la clínica y de los estudios epidemiológicos. Unas y otros nos informan de factores antecedentes, características del consumo y riesgos asociados al mismo que es necesario modificar para alcanzar las metas señaladas.

La elección del origen de los datos de observación no es indiferente. La clínica, puesto que extrae sus datos a partir de las personas que aparecen con el problema, plantea la dificultad de establecer si las características observadas son antecedentes o realmente una consecuencia del consumo y de la dinámica que genera. Por eso mismo, los datos de la clínica nos proporcionan información sobre consecuencias evitables del consumo, y de las secuencias con que se manifiestan.

En cuanto a los estudios sociales y epidemiológicos, muchas veces se limitan a describir cantidades consumidas y frecuencias, y sus relaciones con variables sociodemográficas. Aunque su contribución al conocimiento del problema es interesante, su interés preventivo es más bien limitado. Muchas de estas variables no son modificables, y sólo permiten afinar en la selección de los destinatarios (por ejemplo, sexo y grupos de edad). Mayor interés tienen los estudios que describen características del comportamiento, relaciones o actitudes de consumidores y no consumidores y su entorno. Cuando los estudios son longitudinales, el análisis de estos componentes permite determinar secuencias en la progresión, factores antecedentes, etc. Estos factores se suelen denominar de riesgo y de protección.

El interés por los factores de riesgo procede de una de las últimas evoluciones de la epidemiología, que extendió su objeto de estudio desde la descripción de la enfermedad a los factores que influyen en la misma. El análisis de estos factores y de su evolución constituye hoy uno de los objetos de estudio más importantes para el control de las enfermedades, y su metodología ha trascendido el campo puramente sanitario para alcanzar al conjunto de los comportamientos humanos.

Por lo que concierne al consumo de drogas, se han identificado numerosos factores de riesgo. Algunos de ellos aparecen de manera insistente en la mayor parte de los estudios y parecen bien establecidos. Otros 
de esos factores no parecen tan consistentes, en los diferentes estudios tienen comportamientos distintos, o incluso contradictorios, y quizá están sujetos a la influencia de otras variables (como la edad o el sexo) o están condicionados por otros factores que actúan antes. El lector interesado puede encontrar excelentes resúmenes de esos factores, ordenados por áreas de influencia (Hawkins, Catalano y Miller, 1992; Peterson, Hawkins y Catalano 1992; Moncada, 1997).

\subsection{Cómo integrar los objetivos: el papel de la teoría.}

Los programas con expectativas muy concretas y de alcance limitado, pese a un evidente potencial preventivo, debido a la falta de una teoría integradora, se detienen en un abordaje demasiado parcial del problema. De esta manera, el impacto que tienen como conjunto es más bien escaso. Secuenciar los objetivos en una teoría integradora permitiría introducir en el programa otros componentes que abordarían cada uno unos objetivos desde distintos ámbitos y a través de múltiples intervenciones.

Sin embargo, en su visión de la historia de la prevención, Hansen (1996) distingue tres períodos según el tipo de factores la han dirigido: intuición, teoría y datos. El período orientado por la intuición ha dado lugar a programas del tipo DARE que han tenido escaso o nulo efecto. El período de los programas basados en la teoría ha sido más fructífero, pero, desde su perspectiva, el desarrollo de la ciencia de la prevención requiere el abandono de la orientación teórica y basarse sólo en la evidencia empírica sobre las variables relacionadas con el uso de drogas. Concluye que la prevención ya debería abandonar los métodos que pretenden modificar variables que tienen una débil relación estadística con el uso de drogas.

Una posición semejante podemos encontrar en Gorman (1998), para quien la mayor parte de programas y sus evaluaciones no se basan en la evidencia empírica, e incluso esta evidencia es modificada según los intereses de los promotores de los programas.

Tal vez no hace falta llevar la crítica tan lejos. Es evidente que la teoría no puede estar al margen de los datos y proponer objetivos inútiles. Pero, como se ha visto, el problema no es este. La dificultad no está en que los objetivos sean irrelevantes, sino en que sólo alimentan un proceso que no encuentra continuidad y coherencia con fases posteriores (Martín, 1995).

De ahí el papel que todavía corresponde a la teoría, entendida como conjunto de postulados coherentes entre sí, que describen las relaciones entre variables y explican las causas de estas relaciones.

Los datos de observación sólo adquieren sentido a través de su interpretación. Es la teoría lo que confiere ese sentido, por lo que también es a partir de la teoría que podemos discriminar qué datos son pertinentes y cuáles no, y la teoría orienta de esta manera la observación. También es la teoría lo que nos permite arriesgar hipótesis sobre el comportamiento futuro de algún aspecto de la realidad.

Por eso, la teoría debería tener un papel más central en el campo de las drogodependencias y guiar tanto la observación como las intervenciones preventivas y terapéuticas. Que muchos programas preventivos carezcan de base teórica no es por falta de teorías. En una reciente revisión (Becoña, 1999 a) aparecen descritas veintiuna teorías y modelos de complejidad diversa, seleccionadas por su actualidad, relevancia y apoyo empírico.

Disponer de programas basados en la teoría comporta ventajas tanto para el propio programa como para su evaluación (Becoña, 1999 b).

\subsection{El diseño de las intervenciones.}

Un elemento fundamental en el diseño de las intervenciones preventivas es la elección de sus destinatarios. 
En el contexto de la prevención primaria se han ensayado taxonomías diversas. Catalano y Dooley (1982) distinguen entre prevención primaria proactiva y reactiva.

- Proactiva: estrategias de acción que intentan prevenir la ocurrencia de factores de riesgo.

- Reactiva: estrategias de acción que intentan preparar al sujeto para reaccionar de forma efectiva en las situaciones de riesgo. Cowen (1985) distingue entre:

- Prevención primaria en el ámbito de los sistemas: se dirige a reducir las fuentes o factores de riesgo, e incrementar las oportunidades para vivir de forma adecuada en un contexto social dado.

- Prevención primaria centrada en la persona: un objetivo es aumentar la capacidad de las personas para afrontar eventos y situaciones de riesgo. Esta prevención puede estar:

- Focalizada en la situación: centrada en las personas que sufren situaciones conflictivas y de riesgo, para reducir la probabilidad de que tengan consecuencias negativas.

- Focalizada en el fortalecimiento de las competencias: intenta dotar a grupos todavía no afectados de las competencias y habilidades que refuerzan sus propias capacidades adaptativas.

Un programa preventivo debería incluir estrategias complementarias para modificar el conjunto de factores implicados y alcanzar a la totalidad de la población según sus riesgos respectivos.

\section{OBJETIVOS RESPECTO DEL CONSUMO DE CÁNNABISY SUS CONSECUENCIAS}

La prevención del consumo de drogas no puede plantearse de forma aislada.

El cánnabis es la substancia ilegal usada con más frecuencia. Su consumo se ha estabilizado en las frecuencias altas. Es también la droga que aparece con mayor frecuencia en el policonsumo (ver tabla 1).

\begin{tabular}{|lc|}
\hline $\begin{array}{c}\text { Tabla 1. Frecuencias de policonsumo de } \\
\text { cánnabis con otras substancias }\end{array}$ \\
\hline Combinaciones & $\%$ \\
Con alcohol & 50.6 \\
Con alcohol y éxtasis & 8.4 \\
Con alcohol, éxtasis y cocaína & 7,8 \\
Con alcohol y cocaína & 2.0 \\
\hline & \\
\hline (Calafat y otros, 1999) & \\
\hline
\end{tabular}

Llama la atención el 8,4\% que lo consume con éxtasis, combinación que parece extraña y de la que no se habla mucho.

Los datos indican que no parece justificado emprender programas de prevención específica del consumo de cánnabis, aislados de las otras drogas. Los consumidores de cánnabis no poseen características que les diferencien de los consumidores de otras drogas, al menos en las primeras etapas del consumo. No parece que exista un riesgo específico que predisponga al consumo de cánnabis. Mientras no dispongamos de otras evidencias, hay que pensar que existe un riesgo general de consumo de drogas que se orienta hacia una u otra droga en función de la disponibilidad de cada substancia y de la experiencia previa con otras substancias.

En cualquier caso, lo que sí parece que sería importante es incidir en los riesgos del policonsumo.

2) El primer objetivo es la reducción del consumo de alcohol y tabaco.

El inicio del consumo generalmente se establece con el alcohol o el tabaco. Cuanto antes se empieza con estas drogas, el riesgo de uso de otras substancias es mayor y, además, se incrementa la probabilidad de alcanzar niveles elevados de adicción.

Según muestran los datos, la siguiente droga que hace su aparición es el cánnabis, y bastante más tarde las diversas derivadas de las anfetaminas y de las que se denominan, más propia o impropiamente, drogas de diseño. 


\begin{tabular}{|l|c|c|c|}
\hline \multicolumn{4}{|c|}{ Tabla 2. Edades de inicio de consumo } \\
\hline & MíNIM0 & MÁxIM0 & MEDIA \\
\hline Alcohol & 13,3 & 15,9 & 14,6 \\
Tabaco & 14,1 & 16,0 & 14,8 \\
Cánnabis & 15,2 & 17,1 & 16,0 \\
Anfetaminas & 16,2 & 19,2 & 17,7 \\
LSD & 16,3 & 20,6 & 17,7 \\
Éxtasis & 16,5 & 19,9 & 18,4 \\
Cocaína & 17,7 & 20,7 & 19,3 \\
Heroína & 17,8 & 21,1 & 19,4 \\
\hline \multicolumn{4}{|l|}{} \\
(Calafat y otros, 1999)
\end{tabular}

En un seguimiento de adolescentes a un año y a cuatro años se ha visto que el nivel de uso de tabaco puede predecir el uso de alcohol un año después, y que aquellos que incrementan su consumo de tabaco también aumentan el de alcohol durante los siguientes cuatro años. Pero el uso de tabaco es mejor predictor del uso de cánnabis que de alcohol; los mayores fumadores de tabaco no sólo se manifiestan más fácilmente como consumidores de cánnabis al cabo de un año, sino que también aumentan más rápidamente su consumo de cánnabis en los siguientes cuatro años (Duncan, Duncan y Hops, 1998).

Estos hallazgos son coherentes con la teoría secuencial que sostenemos, basada en el modelo de carreras de desviación de Becker (1963): las conductas desviadas se desarrollan en una secuencia de etapas que se constituyen como itinerarios o carreras de desviación progresiva.

Para la mayor parte de adolescentes, la disponibilidad de una droga aumenta la probabilidad del primer consumo; la probabilidad de repetir el consumo de una droga determinada es más elevada después de un primer consumo experimental: el uso frecuente de una droga de bajo nivel de riesgo percibido (como las drogas legales) aumenta la probabilidad de recibir incitaciones para usar drogas del siguiente nivel y la repetición de incitaciones aumenta la probabilidad del primer consumo; la probabilidad de repetir el consumo de una droga determinada es más elevada después de un primer consumo experimental; el uso frecuente de una droga de bajo nivel de ries- go percibido (como son las drogas legales) aumenta la probabilidad de recibir incitaciones para usar drogas del siguiente nivel; la repetición de estas incitaciones aumenta la probabilidad de que se acepte, etc. (Calafat, Amengual, Monserrat y Farrés, 1983; Calafat, Amengual, Mejías y Borrás, 1989; Amengual, Calafat y Palmer, 1993).

Una primera conclusión útil para la prevención, es que la prevención debe empezar por las drogas legales o, por lo menos, por aquellas que los destinatarios del programa estén más próximos a consumir.

Pero, además, estos datos merecen otras reflexiones.

Es notorio que el consumo de drogas empieza con el alcohol y tabaco, que son drogas legales e institucionalizadas, a unas edades en que está prohibida su venta a los jóvenes. No es, por tanto, el caso de un comercio ilegal y clandestino, sino una práctica que llevan a cabo ciudadanos a los que no se les conoce ningún interés en producir perjuicio a los jóvenes.

Lo que esto pone de manifiesto es que existe un determinado grado de tolerancia familiar y social cuya modificación puede constituir un importante objetivo preventivo, ya sea mediante medidas de refuerzo de la normativa y de vigilancia de un cumplimiento, de apoyo a la función de control familiar, o de fomento de alternativas de uso de tiempo libre y de consumo por parte de los jóvenes.

Por otra parte, un somero análisis de las estrategias publicitarias para incitar al consumo de alcohol y de tabaco nos puede enseñar el papel simbólico que representan en el imaginario colectivo, y el tipo de necesidades que supuestamente están destinadas a cubrir. De esta manera podríamos definir un tercer grupo de objetivos relacionados con los déficits personales que afectan en mayor o menor medida a todas las personas.

3) También conviene explorar el contexto del consumo. A partir de los datos sobre lugares de reunión de los jóvenes, se observa que los usuarios de cánnabis (como los de alco- 
hol) tienden a reunirse significativamente más a menudo en bares, mientras que los consumidores de éxtasis prefieren las discotecas.

De esta manera, los programas de reducción de riesgos tienen mayor probabilidad de alcanzar a su público si se despliegan en estos lugares.

Por otra parte, la motivación principal de los jóvenes para salir de su casa es para encontrarse con los amigos o para escuchar música.

Se deduce, en primer lugar, la importancia primordial del grupo como lugar de intercambio, de socialización, de incorporación de valores y de hábitos diversos. Y esto tiene una importancia tanto para los objetivos como para las estrategias.

No puede esperarse de un programa preventivo apartar al joven de lo que es un medio para la formación de su identidad. Los programas que pretenden hacer del joven un individuo al margen de las tendencias de un grupo de referencia están condenados al fracaso. De lo que se trata, tal vez, es de intentar cambiar la referencia normativa del grupo. Y siempre, aprovechar las fuerzas que pone en funcionamiento la dinámica grupal, a favor de los objetivos preventivos.

Téngase en cuenta, además, que en el ranking de drogas preferidas el cánnabis es la segunda (21,2\%), por detrás del alcohol $(38,2 \%)$ y muy por encima del éxtasis $(11,0 \%)$ y las otras drogas, y según su percepción, es la droga de moda en Europa (Calafat y otros, 1999)..

El atractivo del cánnabis es importante. Pero también sabemos de qué manera arrastra el deseo percibido. El valor que conferimos a un objeto aumenta cuando creemos que los demás lo valoran. Este efecto es bien conocido tanto en economía como en psicología social (el fenómeno de la universalización). El riesgo del consumo no depende sólo de las características de la substancia. También depende de la significación psíquica y social que se le atribuya.
Ya Béjerot (1981) definió la probabilidad de contagio de la conducta como la suma de los factores de susceptibilidad personal y de exposición ambiental. Cuando la exposición es elevada (disponibilidad, ambiente, presión grupal), no es necesario ser demasiado susceptible. Cuando una conducta es adoptada por un número suficiente de personas (o se cree que es así), pasa a ser la conducta vigente.

La disponibilidad de una droga, por su parte, ejerce un importante papel: considerando sólo a los que han usado alguna droga, entre la primera oportunidad $u$ ofrecimiento de consumo y la primera vez que probaron esa droga, transcurrió menos de una año (Van Etten y Anthony, 1999).

Esto nos lleva, por otro camino, el cambio de la percepción normativa como objetivo para la prevención

También cabe destacar, en este apartado, el papel de la música como lazo de unión entre los jóvenes, al tiempo que cada estilo musical permite una identificación particular con un grupo y diferenciarse del resto.

No se ha explorado la posible eficacia de los mensajes musicales. Tal vez el recuerdo de la ñoñería de los curas, frailes y monjas cantores que proliferó en los años sesenta, nos espanta. No obstante, sin caer en ese espectáculo lamentable, parece que una música comprometida con los valores actuales, podría hacer algo más que promover el consumo de cánnabis y otras drogas.

Como se recoge en un artículo aquí mismo (Calafat y Juan, 2000), alrededor del cánnabis existe una cultura pretendidamente alternativa, que se basa en un conjunto de planteamientos ideológicos y místicos de la más variada procedencia. Este cultura sistemáticamente reinterpreta de manera interesada la historia y los datos científicos acerca de la planta, sus derivados y su consumo. Es difícil calcular el potencial económico que la sostiene a través del comercio y la publicidad de grandes empresas. Pero resulta evidente que nos enfrentamos a hechos muy parecidos a los que mantienen el consumo de alcohol y 
de tabaco en los niveles actuales. Cualquier programa preventivo debería tener entre sus principales objetivos contrarrestar los efectos de esta presión al consumo.

4) Las conductas de riesgo también pueden constituir una base para la definición de objetivos.

Ya se ha mencionado el policonsumo (particularmente con alcohol y éxtasis) de los consumidores de cánnabis. Pero hay más. La probabilidad de conducir un vehículo bajo influencia de las bebidas alcohólicas no afecta sólo a los consumidores de alcohol. También es significativamente mayor entre los usuarios de cánnabis. Riesgo que se añade al propio de conducir después del consumo de esa substancia.

La prevención orientada a evitar el uso de vehículos bajo los efectos del alcohol o de drogas es un objetivo prioritario. Máxime cuando sabemos que el uso de drogas está asociado al ocio y que a menudo el disfrute del ocio significa el desplazamiento de un lugar a otro. Una evidencia de este riesgo es que la mayoría (65\%), se desplazan a dos o tres sitios durante la noche, y el $14 \%$ acude a más de cuatro. Los desplazamientos se realizan en el propio vehículo $(35,9 \%)$ o en el de un amigo (28,8\%), siendo minoritario el uso de transporte público (Calafat y otros, 1999), que, además, en muchos lugares no está disponible de noche. Esta frecuencia de desplazamientos combinada con el uso de alcohol y drogas tiene un resultado: los accidentes de tráfico son la primera causa de muerte entre 15 y 24 años, y la segunda, después del SIDA, entre los 25 y 34 años (Martínez y Llácer, 1998).

La percepción del riesgo que implican determinadas conductas disminuye significativamente a medida que el consumo se hace más frecuente. Sin embargo, pese al descrédito de las estrategias basadas en el miedo, se ha podido comprobar que la percepción del riesgo puede ser determinante para reducir la frecuencia de determinados comportamientos, y la aceptación social de los mis- mos. La evidencia procede tanto de los estudios longitudinales, como del seguimiento del uso comunitario de drogas. No tener miedo a las consecuencias del uso de una substancia es un buen predictor del uso posterior de la misma (Petraitis y otros, 1998). También se ha podido encontrar una clara relación entre la percepción de consecuencias dañinas y de desaprobación social del consumo de cánnabis, y la disminución de todas sus frecuencias de uso (Bukoski, 1991).

5) El consumo de cánnabis, como el de otras drogas, está relacionado con determinadas disposiciones personales cuya modificación puede constituir un importante objetivo preventivo.

Los consumidores de frecuencias más altas tienen también una puntuación significativamente mayor en la Escala de Búsqueda de Sensaciones de Zuckerman y una mayor probabilidad de tener comportamientos asociales (Calafat y otros, 1999).

En una revisión de estudios longitudinales aparece una estrecha relación entre uso de substancias ilegales y la vinculación con compañeros que tienen conductas desviadas $y$, en menor grado, desvinculación o rebelión contra las instituciones encargadas de la socialización, como pueden ser la religión, la escuela y la familia (Petraitis y otros, 1998).

En este orden de cosas, también se sabe que la adolescencia representa el proyecto de hacerse adulto. Este proceso no siempre es fácil, y está marcado por diversos intentos o ensayos en el área de la construcción de la identidad, del establecimiento de relaciones y del ejercicio de los distintos roles. La llamada crisis adolescente y muchas de sus manifestaciones deben situarse en este contexto. Pero hay factores que pueden entorpecer el proceso. El uso de cánnabis, y posiblemente el uso de todas las drogas, incrementa el riesgo de adoptar roles marginales o, por lo menos, poco convencionales, como aplazamiento o inestabilidad de las relaciones de pareja, tener hijos antes de asegurar las con- 
diciones para su sostenimiento y paro, subempleo o trabajo marginal. De esta manera, el uso precoz de cánnabis influye negativamente en la capacidad para asumir los roles adultos y prolonga, así la duración de la adolescencia (Brook y otros, 1999).

Según este último estudio, aquellos que asumen más temprano los roles adultos disminuyen el riesgo de uso posterior de cánnabis. Sin embargo, parece que tampoco es conveniente adelantar demasiado el proceso. Implicarse en responsabilidades adultas a una temprana edad en la adolescencia interfiere en la adquisición de las habilidades psicosociales necesarias para el éxito posterior en esos roles (Newcomb, 1996).

\section{PROGRAMAS E INTERVENCIONES}

Para esta revisión se utiliza la nomenclatura propuesta de Gordon (1987), adoptada por el Instituto de Medicina (1994) y el NIDA² estadounidenses, según la cual podemos desarrollar un continuo de actuaciones que llamaremos universales, selectivas e indicadas o prescritas según el riesgo relativo de los participantes.

\subsection{Universales.}

Las intervenciones de tipo universal están destinadas a prevenir los precursores del uso de drogas o la iniciación del uso en poblaciones generales, como jóvenes y familias.

Incluyen campañas en los medios de comunicación y otras técnicas de educación pública. Cuando se aplican en escuelas, se destinan a todos los estudiantes de los cursos a los que van dirigidos. Se basan generalmente en intervenciones cortas o poco intensivas (de 6 a 18 horas). Para su aplicación no suele ser necesario un entrenamiento específico porque se apoyan en materiales altamen- te estructurados. No hace falta tampoco un personal especial. En las escuelas lo aprenden a hacer los propios profesores.

Su coste por participante es relativamente bajo, porque no requieren ninguna parafernalia extra. Por eso se han convertido en el recurso más utilizado, sobre todo en las escuelas. Además, suelen tener una buena relación coste-eficacia.

Uno de los principales problemas es que, en la escuela, aumentan la carga de trabajo del profesorado. Sin embargo, el profesorado puede percibir estos programas como una ayuda para mejorar un trabajo y para que la escuela alcance sus objetivos preventivos.

Otro de sus inconvenientes es que sus resultados son temporales e insuficientes para los jóvenes de algo riesgo y sus familias. En general, aunque tienen resultados positivos, no están a la altura de las expectativas que general, y de ninguna manera hay que pensar que este tipo de oferta cubra todas las necesidades preventivas.

Como se ha mencionado antes, habitualmente se utilizan estrategias de este tipo en los programas escolares y comunitarios, incluyendo las familias.

Algunos programas sólo pretenden mejorar el nivel de conocimientos de sus destinatarios. Tal es, por ejemplo, uno de los escasos proyectos específicos sobre cánnabis que se ha desarrollado en nuestro país, y que ha mejorado significativamente la información de los participantes (Hazkunde, 1999).

Lo habitual, sin embargo, es que los programas aspiren a evitar, reducir, detener o modificar de alguna manera el consumo. Pero no todos son igualmente aptos para ello. La evidencia que se desprende de la revisión de las evaluaciones muestra de una manera muy consistente que los abordajes que se centran de manera exclusiva, o prioritaria, en la transmisión de información, no logran prevenir, reducir o disuadir del consumo de drogas (Botvin, Botvin y Ruchlin,

${ }^{2}$ Ver, por ejemplo, el monográfico editado por Leukefeld y Bukoski (1991) en el que diversos capítulos ya utilizaban esta clasificación. 
1998). De ahí que, sin olvidar que la información tiene un papel importante, haya que ensayar otros enfoques.

Existe una gran diversidad: cognitivo y afectivo, de influencias sociales, de entrenamiento de habilidades personales y sociales, de acompañamiento, de cambio en el clima escolar, de participación comunitaria, y de implicación de padres.

La mayoría de los programas escolares que se utilizan en nuestro país pueden encuadrarse en alguna de estas categorías. Así, entre los más utilizados tenemos el programa A tu salud (Programa de Prevención de Drogodependencias, 1994) que se centra en los riesgos de la adolescencia relacionados con la socialización secundaria; Plan Integral de Prevención Escolar (FAD, 1996), que adapta los objetivos y las intervenciones a los que corresponden a cada ciclo y etapa educativa; Entrenamiento en habilidades de vida, ampliación y adaptación del programa de Botvin (Luengo y otros, 1998); Tú decides (Calafat y otros, 1999), cuyo objetivo final es el aprendizaje de la toma de decisiones en el contexto psicosocial del grupo y de la dinámica adolescente.

Según han mostrado repetidamente Tobler y su equipo (1986, 1992, 1997), el tamaño del efecto de los programas escolares está determinado en primer lugar por la metodología utilizada. La mayor diferencia aparece entre programas interactivos y no interactivos. Los primeros muestra una eficacia mucho mayor que los segundos.

Cabe recordar que los programas interactivos incluyen actividades de grupo entre las técnicas para promover la adquisición de habilidades. En ellos, los participantes ponen en juego sus creencias, actitudes y habilidades, y reciben un feed-back adecuado en una atmósfera de colaboración.

Los programas no interactivos, que muestran escaso o nulo efecto, tienen la forma de enseñanza tradicional, donde el profesor imparte sus lecciones de manera unidireccional, y sólo eventualmente los alumnos participan en discusiones organizadas.
Los programas interactivos suelen tener un enfoque sobre la competencia personal, en vez de sobre los conocimientos. El desarrollo de la competencia personal es una de las tareas que debe realizar el adolescente, y habitualmente realiza el aprendizaje a través de la interacción con los demás, en el seno de uno o varios grupos. Las metodologías que utilizan las técnicas grupales, en la medida que aprovechan los mecanismos que genera el grupo y que favorecen los aprendizajes, muestran resultados superiores.

Además, los adolescentes suelen tener una percepción exagerada del consumo de los demás. Los programas interactivos corrigen esta percepción mediante la confrontación entre iguales, y al desarrollar una percepción más realista, sus ansiedades, la presión a la uniformidad, etc. pueden moderarse. De esta manera también se modifica la definición de la norma, en el doble sentido de norma estadística y de norma de conducta.

Los programas no interactivos no utilizan este potencial y se limitan a una enseñanza que es recibida pasivamente y no tiene capacidad para modificar ni las creencias, ni las actitudes, ni los comportamientos.

Algunos programas han intentado superar esta limitación mediante el uso de técnicas audiovisuales. No es el momento de discutir la eficacia educativa de estas tecnologías. En lo que concierne a la prevención de drogas, un estudio reciente ha mostrado que las técnicas en vivo son significativamente más atractivas que el vídeo, y sugiere que para la enseñanza secundaria sería más efectiva la interacción en vivo que la utilización de vídeos (Miller, Hecht y Stiff, 1998).

Por otra parte, los programas de contenido más amplio son más eficaces que los programas limitados. Los programas que incluyen más de un componente son significativamente más eficaces.

Esta multiplicidad de componentes incluye desde una diversidad de destinatarios: (padres y profesores y entorno comunitario próximo, más que jóvenes solos) a una gradación de objetivos interrelacionados (conoci- 
mientos, influencias sociales, habilidades sociales de rechazo y genéricas, y cambio o refuerzo de normas).

\begin{tabular}{|l|c|c|c|}
\hline \multicolumn{3}{|c|}{ Tabla 3. Efecto relativo de los distintos } \\
programas \\
\hline & POSITIV0 & NEUTRO & NEGATIV0 \\
\hline & & & \\
\hline $\begin{array}{l}\text { Información/clarificación } \\
\text { de valores }\end{array}$ & $30 \%$ & $40 \%$ & $30 \%$ \\
$\begin{array}{l}\text { Educación afectiva } \\
\text { Influencia social } \\
\text { (Por ej.: habilidades de resistencia, } \\
\text { establecimiento de normas, } \\
\text { habilidades sociales ode vida) } \\
\text { Multicomponentes }\end{array}$ & $42 \%$ & $25 \%$ & $33 \%$ \\
\hline (Hansen, 1992) & $72 \%$ & $26 \%$ & $11 \%$ \\
& & $28 \%$ & $0 \%$ \\
\hline
\end{tabular}

Finalmente, es conveniente saber que los programas genéricos suelen ser más eficaces que los que toman una sola droga como objeto, con la excepción de los programas sobre tabaco que se realizan en grupos pequeños. Los programas genéricos incluyen alcohol, tabaco, cánnabis y otras drogas, y su eficacia para cualquiera de ellas es muy superior con los programas interactivos.

\subsection{Selectivos.}

Los programas selectivos están dirigidos sólo a los grupos de riesgo, lo que incluye tanto a los jóvenes como a sus familias. Podrían ser, por ejemplo, los hijos de alcohólicos o de dependientes de drogas; los niños de ambientes con importantes carencias económicas o culturales, muchas veces minorías e inmigrantes; o también los alumnos que han debido cambiar de escuela, a causa de la movilidad de sus familias; e incluso se puede contemplar el uso de anabolizantes entre los atletas.

Para ser eficaces, estos programas deben ser más largos e intensivos, y han de incluir actividades específicamente dirigidos a reducir los riesgos identificados y a reforzar los factores de protección que pudieran estar presentes.

Puesto que sólo participan aquellos que lo necesitan, esta clase de programas puede hacer un uso más eficiente de los escasos recursos especializados en prevención.

Comparativamente, esta clase de programas tiene efectos superiores a los programas universales, pero debido a su alcance más reducido no pueden llegar a ser la elección única de una política preventiva.

También estos programas tienen sus dificultades. Por ejemplo, identificar, reclutar y mantener a los jóvenes en el programa es mucho más difícil que en los programas universales. Además, los criterios de selección pueden derivar en el etiquetado de los participantes, lo que puede tener un efecto negativo en los mismos y en su entorno.

En estos programas también se utilizan técnicas de entrenamiento de habilidades sociales. Véase, por ejemplo, el programa Discover, recientemente adaptado en nuestro país (Díez y otros, 1998). Según un análisis de 75 programas de este tipo realizado en Estados Unidos (Office of Substance Abuse Prevention, 1992), el 84\% ofrecen entrenamiento en habilidades de vida, $81 \%$ habilidades de comunicación, y el 69\% ofrecen entrenamiento en habilidades de resistencia a la influencia social y de los compañeros.

Sin embargo, mientras que los programas universales se basan en principios bien establecidos experimentalmente, los programas selectivos todavía carecen de la suficiente base teórica. Aún así, parece que hay alguna evidencia de que estos programas deben incluir:

- Educación especial, apoyo y entrenamiento de habilidades sociales y de la resiliencia (palabra que hemos incorporado innecesariamente a nuestro idioma, cuando podríamos Ilamarlo simplemente plasticidad) para los jóvenes sometidos a situaciones personales y sociales más vulnerables.

- Ofrecimiento de actividades alternativas para que aquellos que tienen un uso pobre de su tiempo libre o están desocupados.

- Implicación y participación en proyectos comunitarios y cooperativos.

- Tutorías y refuerzo escolar para los que corren riesgo o han sufrido fracaso escolar. 
- Actividades de refuerzo de las culturas minoritarias, y de intercambio multicultural para inmigrantes y sus entornos de acogida.

- Programas de rituales de paso, de apoyo entre iguales o de asesoramiento de jóvenes para incrementar la responsabilidad y el control interno.

- Entrenamiento en habilidades parentales para familias con baja implicación y supervisión, o desbordadas por las dificultades de sus hijos.

Desgraciadamente, hay poco que decir sobre la eficacia de estos programas. En parte, por su historia reciente, y en parte porque la mayoría de los mismos se desarrollan mediante diseños no experimentales y como respuesta inmediata y no programada a las necesidades que aparecen. Pero, dado el interés que despiertan entre los profesionales y entre los responsables locales de las políticas sociales, es de prever un incremento notable. Hay que desear lo que en su diseño incorporen los necesarios enfoques teóricos que justifiquen los objetivos que se persigan, y las previsiones necesarias para su evaluación, sin lo cual poco avanzaremos.

\subsection{Indicados.}

Los programas indicados o prescritos (en el sentido de recetados) están destinados a las personas que manifiestan determinados factores de riesgo identificados: malos resultados escolares, abandono de la escuela, asociación con compañeros que tienen conductas antisociales, o agresividad y trastornos de la conducta.

En general, podemos considerar de alto riesgo a las personas expuestas a muchos riesgos, o que manifiestan un elevado nivel en un solo riesgo.

Estos programas no están destinados sólo a adolescentes y jóvenes. También pueden ser convenientes para determinados alumnos de la escuela primaria, según el factor de riesgo que consideremos. Se sabe que un mismo factor puede tener distintos efectos según la etapa de desarrollo del sujeto. Por ejemplo, la relación con compañeros que tienen conductas antisociales es un factor de riesgo en la etapa de transición de la infancia a la adolescencia, pero no durante la adolescencia misma. Al mismo tiempo, el rechazo de los compañeros, las conductas agresivas y un bajo control familiar en la infancia aumenta la probabilidad de que en la pubertad se elijan compañeros con conductas desviadas (Dishion, 1990).

Según este ejemplo, la prevención de la conducta antisocial en la adolescencia requerirá actuar sobre un tipo de objetivos en la infancia, y sobre otros en la pubertad. Además, no todos los objetivos de modificación de los factores de riesgo implicados pueden alcanzarse con intervenciones aisladas o desde un solo sector o en un solo ámbito.

Los factores de riesgo o de protección individuales, familiares, escolares, de relaciones sociales y del entorno comunitario son interdependientes. Por eso la estrategia preventiva sólo se puede plantear como una multiplicidad de intervenciones sobre cada factor detectado, y desde una perspectiva multisectorial de integración (o por lo menos coordinación) de servicios.

Catalano y colaboradores (1998) plantean dos cuestiones que hay que considerar antes de intervenir con poblaciones de alto riesgo.

La primera es cómo llegar a los individuos en riesgo. Una manera es tomar poblaciones enteras, en las que convivirán personas con bajo y alto riesgo en proporciones variables (como en las intervenciones de tipo universal). Otra manera es seleccionar a los individuos que tienen ese riesgo particular, lo que entraña un posible etiquetado de esas personas que les resulte perjudicial a medio o largo plazo. Este peligro queda minimizado cuando el riesgo constituye un problema en sí mismo que requiere tratamiento: fracaso escolar, familias conflictivas, abusos o negligencias familiares, etc.

También pueden seleccionarse zonas geográficas definidas como de alto riesgo, y utilizar en ellas procedimientos del tipo universal. 
En estos casos se pueden beneficiar de la intervención tanto los sujetos de bajo como de alto riesgo (Hawkins y otros, 1992). Sin embargo, hay evidencias contradictorias que invitan a tomar precauciones.

En una intervención con adolescentes delincuentes ${ }^{3}$ se comparó la eficacia del grupo de delincuentes solos con la de un grupo mixto. El grupo de delincuentes y no delincuentes juntos resultó ser más eficaz para prevenir reincidencias que el grupo de delincuentes solos. Además, los no delincuentes no se viero a penas afectados por su exposición a modelos delincuentes.

Como contrapartida, Dishion y Andrews (1995) informan de que en los grupos mixtos, si el conductor del grupo carece de entrenamiento suficiente en el manejo de las normas y conductas grupales, se da un efecto de contagio, es decir, se provocan efectos contraproducentes o se reduce la eficacia de la intervención. Por eso es necesario que los profesionales que deban enfrentarse a grupos mixtos, de bajo y alto riesgo, tengan una amplia formación y experiencia en el trabajo grupal, y cuenten además con una buena supervisión.

La segunda consideración es que los factores de riesgo se pueden usar sólo para seleccionar a los sujetos de alto riesgo, o también como objetivo de la intervención. Usarlos sólo como criterio de selección de los sujetos incrementa la probabilidad de ocasionar nuevos problemas al grupo a causa del etiquetado. Por eso es aconsejable que los factores de riesgo constituyan también un objetivo de la intervención, es decir, sólo se deberían constituir grupos formados a partir de unos factores de riesgo determinados cuando estos factores requieran una intervención específica.

Dentro de este tipo de programas podemos encuadrar las intervenciones que tienen como objetivo la reducción del daño. Estas intervenciones, dirigidas a los usuarios de drogas, pretenden minimizar algunas de las consecuencias adversas del consumo de drogas: policonsumo, conducción de vehículos, sobredosis, reacciones negativas, etc.

Algunos tienen como destinatarios a los usuarios de discotecas y centros de diversión, a grupos informales que se reúnen en la calle, absentistas escolares, etc. Su objetivo es claramente informativo y no persiguen en absoluto otras modificaciones de actitudes y comportamientos más difíciles de alcanzar en estos grupos.

La dificultad mayor estriba en que el mensaje llegue a sus destinatarios. Se han ensayado los procedimientos más variopintos, desde folletos, a la acción directa de compañeros informadores. Casi siempre se incluye un teléfono de información o de emergencia. Últimamente, además, se ofrece información o intercambios a través de internet.

Puesto que el objetivo es persuadir a los destinatarios de que adopten determinadas precauciones, lógicamente deben utilizarse técnicas de marketing social, donde los medios, la brevedad y claridad del mensaje, y el atractivo estético son esenciales.

Tampoco en este tipo de programas hay evaluaciones de eficacia, si exceptuamos medidas indirectas como la evolución de las cifras de accidentes que queremos evitar. Es más frecuente el recuento de material distribuido y el feed-back que se recoge a través de los contactos y las llamadas a los teléfonos de información.

\section{A MODO DE CONCLUSIÓN. ALGO MÁS SOBRE LA EFICACIA}

A lo largo de este artículo se ha pretendido mostrar un esbozo de lo que debería constituir un programa preventivo y de los objetivos que debería perseguir.

${ }^{3}$ Feldman, R.A. y Caplinger, T.E. "The St.Louis Experiment: Treatment of antisocial youths in prosocial peer groups." Ponencia presentada a la American Society of Criminology, Toronto, 6 de noviembre de 1982 (citado por Catalano y otros, 1998). 
Debemos reconocer que hoy por hoy los resultados generales de la prevención son, en su conjunto, bastante decepcionantes. Si exceptuamos algunos años a finales de la década de los ochenta y principios de los noventa, el consumo de drogas, y especialmente de cánnabis, no ha dejado de aumentar. Eso ha ocurrido, paradójicamente, cuando se destinan a la prevención más recursos que nunca.

Se puede argumentar que los recursos todavía no son suficientes y que se deberían aumentar. También podemos creer que la prevención debe redoblarse, porque cada día se añaden nuevos factores que incitan al consumo.

Todo eso es, posiblemente, cierto. Pero también es preciso admitir que algunas veces los programas preventivos no se basan en ninguna evidencia empírica, tampoco proceden de ninguna reflexión teórica, ni tienen una planificación adecuada.

Los programas que funcionan no son necesariamente los más aceptados. Como se ha pretendido mostrar en estas páginas, los mejores programas suelen ser aquellos que se basan en una metodología experimental, que seleccionan un conjunto amplio y coherente de objetivos, y que movilizan diversos recursos en un plan de intervención que se mantiene en el tiempo. Esto significa integrar actuaciones y servicios en la escuela, en la familia y necesariamente también en todos los ámbitos comunitarios (asociacionismo, tiempo libre, servicios personales, etc.). Sólo de esta manera se puede conseguir que la intervención alcance a la mayor parte de los factores de riesgo y de protección.

Pero eso no se puede lograr de manera aislada. Los que trabajamos en prevención deberíamos asumir que nuestro trabajo forma parte de un esfuerzo conjunto y en un marco teórico determinado. Aunque es necesario que cualquier programa se adapte a cada contexto en que se vaya a aplicar, también se puede advertir que la actual dispersión no se corresponde siempre con esta necesidad.
Quizá ya ha pasado el tiempo de diseñar intervenciones aisladas y limitadas. Sería más fructífero que cada nueva actividad, después de la pertinente evaluación, se pudiese integrar en un discurso teórico compartido, y formar parte del conjunto de actividades e intervenciones que constituyen los programas más amplios.

Para ello es necesaria una información abierta y accesible, y promover un debate amplio sobre los principios generales de la prevención y de su aplicación concreta. Que cada uno pueda saber en qué están los demás, y ofrecerles a su vez los propios hallazgos. Quizá de esta manera se lograría una mejora sustancial de la calidad de los programas, se rentabilizarían los recursos y se alcanzarían mejores resultados.

\section{BIBLIOGRAFÍA}

Amengual, M.; Calafat, A. y Palmer, A. (1993). Alcohol, tabaco y drogas en enseñanza media. 1981-1988-1992. Adicciones 5 (2), pp. 141-161.

Becoña, E. (1999 a). Bases teóricas que sustentan los programas de prevención de drogas. Plan Nacional sobre Drogas. Madrid.

Becoña, E. (1999 b). Teorías y modelos explicativos en la prevención de las drogodependencias. Idea-Prevención, 19, pp. 62-80.

Becker, H. (1963). Outsiders. MacMillan. New York.

Botvin, G.J.; Botvin, E.M. y Ruchlin, H. (1998). School-based approaches to drug abuse prevention: evidence for effectiveness and suggestions for determining cost-effectiveness. En: Bukoski, W.J y Evans, R.I. (eds.). Costbenefit/cost-effectiveness research of drug abuse prevention: implications for programming and policy. National Institute on Drug Abuse. Rockville. pp. 59-82.

Brook, J.; Richter, L.; Whiteman, M. y Cohen, P. (1999). Consequences of adolescent marihuana use: incompatibility with the assumption of adult roles. Genet Soc Gen Psychol Monogr, 125 (2), pp. 193-207.

Bukoski, W.J. (1991). A framework for drug abuse prevention research. En: Leukefeld, C.G. y Bukoski, W.J. (eds.). Drug abuse prevention 
intervention research: methodological issues. National Institute on Drug Abuse. Rockville. pp. 7-28. (Traducción española: Estudios sobre intervenciones en prevención del abuso de drogas: aspectos metodológicos. CEPS, Madrid, 1995. pp. 11-31).

Calafat, A. (1995). Los factores de riesgo como fundamentos de programas preventivos. En: Becoña, E.; Rodríguez, A. y Salazar, I. (coords.). Drogodependencias. IV. Prevención. Servicio de Publicaciones de la Universidad de Santiago de Compostela. pp. 75-103.

Calafat, A.; Amengual, M.; Farrés, C. y Monserrat, M. (1983): Características del consumo de alcohol, tabaco y otras drogas entre alumnos de Enseñanza Media de Mallorca. Drogalcohol VIII (4), pp. 155-174.

Calafat, A.; Amengual, M.; Mejías, G. y Borrás, M. (1989): Consumo de drogas en enseñanza media. Comparación entre 1981 y 1988. Revista Española de Drogodependencias 14 (1), pp. 9-28.

Calafat, A.; Amengual, M.; Farrés, C. Mejías, G. y Borrás, M. (1999). Tú decides. Programa de educación sobre drogas. Consell Insular de Mallorca. Equip d'Atenció y Prevenció Comunitària. Promoció de la Salut. Palma de Mallorca ( $4^{a}$ edición revisada y actualizada).

Calafat, A.; Bohrn, K.; Juan, M.; Kokkevi, A.; Maalsté, N.; Mendes, F.; Palmer, A.; Sherlock, K.; Simon, J.; Stocco, P.; Sureda, M.P.; Tossmann, P.; Wijngaart, G. y Zavatti, P. (1999). Night life in Europe and recreative drug use. Sonar 98. IREFREA. Palma de Mallorca.

Calafat, A.; Juan, M.; Becoña, E.; Fernández, C.; Gil, E.; Llopis, J.J. En este volumen pp.225-267.

Catalano, R.A. y Dooley, D. (1982). Economic change in primary prevention. En: Price, R.H.; Ketterer, R.F.; Bader, B.C. y Monahan, J. (comps.). Prevention in mental health. Research, policy and practice. Sage. Beverly Hills.

Catalano, R.F.; Haggerty, K.P.; Gainey, R.R.; Hoppe, M.J. y Brewer, D.D. (1998). Effectiveness of prevention interventions with youth at high risk of drug abuse. En: Bukoski, W.J y Evans, R.I. (eds.). Cost-benefit/cost-effectiveness research of drug abuse prevention: implications for programming and policy. National Institute on Drug Abuse. Rockville. pp. 83-110.

Comité Européen de Santé Publique (1981). Comité restreint d'experts sur la prévention dela toxicomanie. Rapport Final. Conseil de I'Europe. Estrasburgo.

Cowen, E.L. (1985). Person.centered approaches to primary prevention in mental health: situationfocused and competence enhancement. American Journal of Community Psychology, 13, pp. 31-48.

Díez, F.; Varlea, B.; Salinas, F.; Fuentes, P.; Cortés, I.; Domínguez, A. y Rodríguez, M. (1998). Deva. Desarrollo de valores y autoestima. IPE. Caritas Española. Bilbao.

Dishion, T.J. (1990). The peer context of troublesome behavior in children and adolescents. En: Leone, P. (ed.): Understanding troubled and troublesome youth. Sage. Beverly Hills. pp. 128-153.

Dishion, T.J. y Andrews, D.W. (1995). Prevention escalation in problem behaviors with high-risk young adolescents: immediate and 1-year outcomes. Journal of consulting and Clinical Psychology, 63, pp. 538-548.

Duncan, S.C., Duncan, T.E. y Hops, H. (1998). Progressions of alcohol, cigarette and marijuana use in adolescence. Journal of Behavioral Medicine, 21, pp. 375-388.

European Monitoring Centre for Drugs and Drug Addiction (1999). 1999 Extended annual report on the state of drugs problem in the European Union. Office for Offficial Publications of the European Communities. Luxemburg.

Fundación de Ayuda contra la Drogadicción (1996). Plan Integral de Prevención Escolar (PIPES). FAD. Madrid.

Gilchrist, L.D. (1991). Defining the intervention and the target population. En: Leukefeld, C.G. y Bukoski, W.J. (eds.). Drug abuse prevention intervention research: methodological issues. National Institute on Drug Abuse. Rockville. pp. 110-122. (Traducción española: Estudios sobre intervenciones en prevención del abuso de drogas: aspectos metodológicos. CEPS, Madrid, 1995. pp. 109-120).

Gordon, R. (1987). An operational classification of disease prevention. En: Steinberg, J.A. y Silverman, M.M. (eds.). Preventing mental disorder. Department of Health and Human Services. Rockville. pp. 20-26.

Gorman, D.M. (1998). The irrelevance of evidence in the development of school-based drug prevention policy, 1986-1996. Evaluation Review, 22 (1), pp. 118-146. 
Hansen, W.B. (1992). School-based substance abuse prevention: a review of the state of the art curriculum, 1980-1990. Health Education Research, 7 (3), pp. 403-430.

Hansen, W.B. (1996). Prevention programs: What are the critical factors that spell success? National Conference on Drug Abuse Prevention Research: presentations, papers and recommendations. Washington, september19-20. National Clearinghouse for Alcohol and Drug Information. Rockville.

Hawkins, J.D.; Catalano, R.F. y Miller, J.Y. (1992). Risk and protective factors for alcohol and other drug problems in adolescence and early adulthood: implications for substance abuse prevention. Psychological Bulletin, 112, pp. 64105.

Hawkins, J.D.; Lishner, D.M. y Catalano, R.F. (1985). Childhood predictors and the prevention of adolescent substance abuse. En: Jones, C.L. y Battjes, R.J. (eds.). Etiology of drug abuse: implications for prevention. National Institute on Drug Abuse. Rockville. pp. 75-126.

Hazkunde (1999). Prevención sobre el consumno de cannabis dirigido a jóvenes mayores de 16 años de la C.A.V. Evaluación del proyecto piloto. (En elaboración).

Institute of Medicine. Committee on Prevention of Mental Disorders (1994). Reducing risks for mental disorders: frontiers for preventive intervention resarch. National Academy Press. Washington.

Kandel, D.B. (1978). Longitudinal research on drug use: empirical findings and methodological issues. Hemisphere. Washington.

Kandel, D.B. (1982). Epidemiological and psychosocial perspectives on adolescent drug use. Journal American Academic Clinique Psychiatry 21 (4) pp. 328-347.

Leukefeld, C.G. y Bukoski, W.J. eds.(1991). Drug abuse prevention intervention research: methodological issues. National Institute on Drug Abuse. Rockville. pp. 57-80. (Traducción española: Estudios sobre intervenciones en prevención del abuso de drogas: aspectos metodológicos. CEPS, Madrid, 1995.

Luengo, M ${ }^{a}$ A.; Otero, J.M.; Romero, E.; Gómez, J.A. y Garra, A. (1998). Entrenamiento en habilidades de vidad. Ampliación y adaptación de programa de J.G. Botvin. Ministerios de Educación y Cultura, de Sanidad y Consumo y del Interior. Universidad de Sanitago de Compostela.
Martín, E. (1995). De los objetivos de la prevención a la "prevención por objetivos". En: Becoña, E. Rodríguez, A y Salazar, I. (coords.). Drogodependencias. IV. Prevención. Servicio de Publicaciones de la Universidad de Santiago de Compostela. pp. 51-74.

Martínez de Aragón, M.V. y Llácer, A. (1998). Mortalidad en España en 1995. Mortalidad general y principales causas de muerte por grupos de edad. Boletín Epidemiológico Semanal, 6 (12), pp. 117-124.

Miller, M.; Hecht, M. y Stiff, J. (1998). An exploratory measurement of engagement of live and film media. Journal of the Illinois Speech and Theatre Association, 49, pp. 69-83.

Moncada, S. (1997). Factores de riesgo y de protección en el consumo de drogas. En: Plan Nacional sobre Drogas. Prevención de las drogodependencias. Análisis y propuestas de actuación. Plan Nacional sobre Drogas. Madrid. pp. 85-101.

National Institute on Drug Abuse (1999). Sixth triennial report to Congress. National Institute of Health. Bethesda.

Newcomb, M.D. (1996). Pseudomaturity among adolescents: construct validation, sex differences, and associations in adulthood. Journal of Drug Issues, 26, pp. 477-504.

Observatorio Español sobre Drogas (1999). Infor$m e n^{\circ} 2$. Ministerio del Interior. Delegación del Gobierno para el Plan Nacional sobre Drogas. Madrid.

Office of Substance Abuse Prevention (1992). Signs of effectiveness: the high-risk youth demonstration grants. US Government Printing Office. Rockville.

Peterson, P.L.; Hawkins, J.D. y Catalano, R.F. (1992). Evaluating comprehensive community drug risk reduction interventions. Design challenges and recommendations. Evaluation Review, 16, pp. 579-602.

Petraitis, J.; Flay, B.R., Miller, T.Q., Torpy, E.J. y Greiner, B.(1998). Illicit substance use among adolescents: a matrix of prospective predictors. Substance Use \& Misuse, 33 (13), pp. 25612604.

Programa de Prevención de Drogas de la Comunidad de Madrid (1994). A tu salud. Ministerio de Educación y Ciencia. Comunidad de Madrid. Ayuntamiento de Madrid. 
Tobler, N.S. (1986). Meta-analysis of 143 adolescent drug prevention programs: quantitative outcome results of program participants compared to a control of comparison group. Journal of Drug Issues, 16 (4), pp. 537-567.

Tobler, N.S. (1992). Meta-analysis of adolescent drug prevention programs: final report. National Institute on Drug Abuse. Rockville.

Tobler, N.S. (1997). Meta-analysis of drug adolescent programs: results of the 1993 meta-analysis. En: Bukoski, W.J. (ed.). Meta-analysis of drug abuse prevention programs. National Institute on Drug Abuse. Rockville.

Tobler, N.S. y Stratton, H.H. (1997). Effectiveness of school-based drug prevention programs: a meta-analysis of research. Journal of Primary Prevention, 18 (1), pp. 71-128.

Van Etten, M.L. y Anthony, J.C. (1999). Comparative epidemiology of initial drug opportunities and transitions to first use: marijuana, cocaine, hallucinogens and heroin. Drug and Alcohol Dependence, 54 (2), pp. 117-125. 